\title{
Mixed Integer Programming Approaches to Planning and Scheduling in Electronics Supply Chains
}

\author{
Tadeusz Sawik*
}

\begin{abstract}
This paper discusses mixed-integer programming (MIP) approaches to planning and scheduling in electronics supply chains. First, the short-term detailed scheduling of wafer fabrication in semiconductor manufacturing and detailed scheduling of printed wiring board assembly in surface mount technology lines are discussed. Then, the medium-term aggregate production planning in a production/assembly facility of a consumer electronics supply chain is described; and finally, the coordinated aggregate planning and scheduling of the manufacturing and supply of parts and production of the finished products is presented. The decision variables are defined, and the MIP modeling frameworks are provided. Two decisionmaking approaches are discussed and compared: an integrated (simultaneous) approach, in which all required decisions are made simultaneously using a complex, large monolithic MIP model; and a hierarchical (sequential) approach, in which the required decisions are made successively using the hierarchies of simpler and smaller-sized MIP models. The paper also highlights the research on stochastic MIP applications to the planning and scheduling in electronics supply chains with disrupted material and information flows due to natural or man-made disasters.
\end{abstract}

Keywords: electronics supply chains, planning and scheduling, mixed integer programming

Mathematics Subject Classification: 90C11, 90B55, 91B30

Submitted: February 14, 2017

Revised: June 01, 2017

\section{INTRODUCTION}

Mixed integer programming (MIP), which has been widely used for long- and mediumterm planning, can also be efficiently used for the short- and medium-term scheduling decision-making in electronics supply chains; e.g., Sawik (2011). The three main stages of an electronics supply chain are wafer fabrication (where integrated circuits are manufactured), surface mount technology (SMT) lines (where printed wiring boards are assembled), and finished-product assembly facilities (Fig. 1). A typical supply chain in the electronics industry may consist of a number of component manufacturers at several locations and one or more producers, where components supplied by the

* AGH University of Science and Technology, Department of Operations Research, Kraków, Poland, e-mail: ghsawik@cyf-kr.edu.pl 
manufacturers are assembled into finished products and ultimately distributed to customers to meet their demand. In such supply chains, productivity may vary from plant to plant, and transportation time and cost are not negligible. Owing to a limited capacity of suppliers, producers, and providers of transportation services, the supply schedules of components and production and distribution schedules of the finished products should be coordinated in an efficient manner to achieve a high customer service level at a low cost. The purpose of supply-chain planning and scheduling is to optimize short- to medium-term tasks and resource allocation in supply chains over a time horizon, considering the trade-off between cost minimization or profit maximization and customer satisfaction or customer service level maximization.

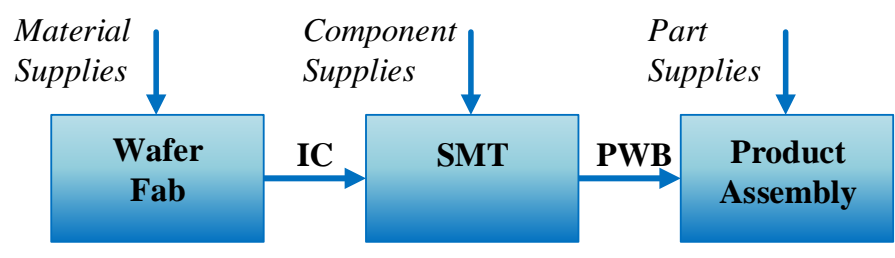

Fig. 1. Electronics supply chain

Short-term supply-chain scheduling is typically concerned with the allocation of tasks and resources of a single facility and with detailed sequencing and timing decisions over a short time horizon (e.g., a shift or day) to complete a given subset of jobs in such a way that one or more job-completion time-related objectives are minimized. A typical single facility considered in the supply chain is a single stage set of parallel machines or a multi-stage flow shop or job shop with single or parallel machines. In contrast, medium-term supply-chain planning deals with the allocation of the tasks and resources of one or more interconnected facilities over a longer time horizon to complete a set of customer orders for the finished products in such a way that the customer service level is maximized and one or more cost objectives are minimized. The typical short-term operational and medium-term tactical decision-making processes connected with electronics supply-chain scheduling are briefly described below.

1) Short-Term Operational Decision-Making:

- Scheduling of semiconductor wafer fabrication.

- Scheduling of printed wiring board assembly.

2) Medium-Term Tactical Decision-Making:

- Aggregate production planning.

- Coordinated planning of material supplies, and production and distribution of products.

Two main decision approaches can be applied (e.g., Sawik, 2011):

Integrated (simultaneous) approach, in which all required decisions are made simultaneously using a complex, large monolithic MIP model;

Hierarchical (sequential) approach, in which the required decisions are made successively, using hierarchies of simpler and smaller-sized MIP models. 
While the integrated approach contributes to the complexity of the underlying mathematical models and decision-making procedures, it is capable of reaching a coordinated solution and global optimum for the entire supply-chain scheduling problem. The hierarchical approach does not guarantee that the overall optimal solution will be attained. In contrast, the complexity of the hierarchical approach can be significantly reduced. In order to model different scheduling problems using MIP approaches, the following three types of decision variables are required:

Binary variables, such as assignment variables (e.g., machine assignment), timeindexed assignment variables (e.g., order to time period assignment), selection variables (e.g., supplier selection), sequencing variables (e.g., precedence variables), etc.

Integer variables, such as number of components, number of machines, number of workers, etc.

Continuous variables, such as timing variables (e.g., completion time of customer order), lot-sizing variables (e.g., production or transportation lot), fractional allocation variables (e.g., allocation of demand for components among suppliers), cost variables, etc.

This paper focuses on MIP application to short-term detailed scheduling and medium-term aggregate planning in electronics supply chains. The coordinated scheduling of manufacturing, supply of components, and assembly and distribution of the finished products are discussed. Furthermore, the MIP approaches can be used for the inter-temporal coordination of medium- and short-term planning and scheduling as well as for the spatial coordination of the producer's and suppliers' schedules. Due to the great variety of planning and scheduling problems in electronics supply chains and the associated MIP formulations, no detailed mathematical models have been presented. The paper rather focuses on a description of the general structures of MIP models and refers the reader to the literature. The remainder of this paper is divided into three main sections. Section 2 addresses the short-term detailed scheduling of semiconductor manufacturing (wafer fabrication) and printed wiring board assembly in surface mount technology lines. Section 3 concerns medium-term aggregate production planning and coordinated supply and production planning. Section 4 briefly describes recent research trends in supply-chain planning and scheduling under disruption risks. The conclusions are presented in Section 5 .

\section{SHORT-TERM DETAILED SCHEDULING}

\subsection{SCHEDULING OF SEMICONDUCTOR WAFER FABRICATION}

In electronics supply chains, the most-complicated stage is the semiconductor manufacturing process. The process consists of four basic phases: wafer fabrication, wafer probe, integrated circuit packaging, and final testing. The most-complex and costly process is wafer fabrication (fab); e.g., Mönch et al. (2012). Wafers are thin discs made of silicon or gallium arsenide, typically $200 \mathrm{~mm}$ to $300 \mathrm{~mm}$ in diameter. Up to several thousand identical chips can be made in each wafer by building up electronic circuits 
layer-by-layer (up to 40 layers for the most-advanced technology). The wafer fab may require up to 700 single process steps and up to three months to complete production. A total of several hundred very expensive machines (ranging in price from a couple hundred thousand dollars to over US $\$ 40$ million per machine) can be found in most wafer fabs. Typical auxiliary resources used in wafer fabs are photo masks (reticles); i.e., carriers of an integrated circuit pattern, which are used in the photolithography process. They are very expensive, and their numbers are often limited. The wafer fab is usually viewed as a reentrant job shop with parallel machines, sequence dependent setups, batch processing, and additional intermediate constraints such as no-wait, limited-wait, no-store, or limited-store. Since one third of all operations in wafer fab are performed on batch processing machines, batch scheduling on parallel machines becomes an important scheduling problem in semiconductor manufacturing. Batch processing requires that each batch of wafers is simultaneously processed on the same machine. Batches of wafers go through repeated sequences of operations, including diffusion, photolithography, etching, ion implanting, etc., to build up successive layers of circuitry on the wafers. For example, diffusion and oxidation machines in wafer fabs are parallel batch machines with incompatible job families. The problem of scheduling on parallel batch machines can be modeled using an MIP approach; e.g., Bilyk et al. (2014). In order to schedule batches of wafers on diffusion and oxidation parallel machines, the following three decisions are made using the MIP approach:

Batch formation, where limited size batches of the same family wafers with identical ready times are formed.

Batch to machine assignment, where each batch is assigned to exactly one parallel machine.

Batch sequencing, where a sequence of batches is determined for each machine.

Owing to the growing demand for Application Specific Integrated Circuits (ASIC) and specialty processors as well as the increasing importance of on-time delivery, meeting one's due dates becomes an important objective in scheduling low-volume, high-variety, and made-to-order wafer fabs. Due to the great complexity of wafer fab scheduling, the use of the exact MIP approaches has become prohibitive for solving realworld fab scheduling problems to optimality in a reasonable computational time. The most-popular approaches are dispatching rules (e.g., Chiang and Fu, 2012), successfully applied in practice by major electronics manufacturers (e.g., IBM, Motorola, Samsung, and Siemens). Nevertheless, the exact MIP formulation can be combined with heuristic procedures to provide a flexible approach for solving large-scale problems. For example, in Bilyk et al. (2014), an MIP model and meta heuristics were applied for scheduling on parallel batch machines to minimize total weighted tardiness as a due-date meeting measure. An MIP-based approach was also applied for scheduling a critical process in semiconductor manufacturing, called an automated wet-etch process (Aguirre et al., 2012). The automated wet-etch station is a complex system that involves a series of successive stages of chemical and water baths as well as a shared automated batch transfer device (such as a robot) used for transferring batches of wafers between consecutive baths. The exact exposure time in chemical baths must be strictly fulfilled, 
and a minimum residence time is enforced in water baths. Thus, no-wait and nostore intermediate storage constraints must be ensured. The resulting problem is a multi-stage flowshop batch scheduling with strict intermediate storage policies in each stage and a single shared resource (robot) with a finite capacity for product movement. An exact MIP-based method was applied to sequentially generate and improve a detailed schedule of production and transfer operations, ensuring strict intermediate storage policies. A decomposition strategy was proposed that combines a rigorous continuous-time MIP formulation with the flexibility of constructive and improvement-based heuristic procedures.

\subsection{SCHEDULING OF PRINTED WIRING BOARDS ASSEMBLY}

In the manufacture of printed wiring boards, Surface Mount Technology (SMT) has been widely used over the last decade; e.g., Sawik (2011). An SMT assembly involves the following basic processes: screen printing of solder paste on a bare board, automated placement of small components, robotic or manual placement of large components, and solder reflow. A typical SMT line consists of several assembly stations in series and/or in parallel, separated by finite intermediate buffers. A conveyor system transfers the boards between the stations. For the process of solder printing and reflow soldering, one machine per line is needed. The number of machines for the placement process can vary, depending on the number and type of components on the boards to be assembled. Various configurations of SMT lines can be encountered in electronics assembly. For example, there are single-pass lines where one pass through the line is required to complete a board or double-pass reentrant lines, where the double-sided boards run twice through the same line; first to assemble the bottom side, and then to assemble the top side.

An SMT line is a practical example of a flexible flow shop. The line typically produces several different board types. Each board must be processed by at most one machine in each stage because of the different routing for different board types. A board which has completed processing on a machine in some stage is transferred either directly to an available machine in the next stage or to a buffer ahead of that stage.

The two major short-term planning problems in SMT assembly are loading and scheduling (Sawik, 2001, 2002). Given a mix of boards to be produced, the objective of the loading problem is to allocate assembly tasks and component feeders among placement stations with a limited working space (so as to balance the station workloads).

In contrast, the objective of the scheduling problem is to determine the detailed sequencing and timing of all assembly tasks for each individual board so as to maximize the line's productivity, which may be defined in terms of throughput or the assembly schedule length (makespan) for a mix of board types.

The limited intermediate buffers between stations result in a blocking scheduling problem where a completed board may remain on a machine and block it until a downstream machine becomes available. This prevents another board from being processed on the blocked machine. In the MIP model, buffers are viewed as machines with zero processing times, and both buffers and machines are referred to as processors. 
In order to schedule the assembly of printed wiring boards in SMT lines, the following three decisions are made using the MIP approach:

Board to processor assignment, where each board is assigned to exactly one parallel processor.

Board timing, where completion time in each stage and departure time from each stage are determined for each board.

Board sequencing, where the sequence of boards is determined for each processor.

The generic MIP model for SMT line scheduling has the following structure; e.g., Sawik (2001):

\section{Minimize Maximum completion time subject to}

1) Assignment constraints for stages with parallel processors to ensure that each board is assigned to exactly one processor and remains on the same conveyor until a shuttle stage.

2) Board completion constraints to ensure that each board is processed at all stages of its processing route.

3) Board departure constraints to ensure that each board cannot be departed from a stage until it is completed in this stage, and to ensure that each board leaves the line as soon as it is completed in the last stage.

4) Board non-interference constraints to ensure that no two boards are simultaneously processed by the same processor.

5) No-buffering constraints to ensure that the processing of each board at each stage starts immediately after its departure from the previous stage.

6) Maximum completion time constraints to define the latest completion time of some board in the last stage; i.e., the makespan of a given production schedule.

The exact MIP approach benefits from some characteristics of the SMT assembly. The most-important features that were exploited in the MIP formulations that directly reduce the required computational effort are:

- a single station at the beginning and at the end of an SMT line,

- at most two parallel placement machines in stages with parallel stations,

- batch-scheduling mode, where boards of one type are consecutively processed,

- fewer than ten different types of boards (batches of boards) to be simultaneously scheduled,

- similar processing times required for different types of boards.

In practice, the similar processing times for different types of boards are typical for all processing stages except for the placement stages. At the placement stages, however, similar processing times are often obtained through the balancing decisions of an SMT line (see, Sawik, 2002). As a result, total placement times for different types of boards at each placement stage tend to be equalized. 


\section{MEDIUM-TERM AGGREGATE PLANNING}

\subsection{AGGREGATE PRODUCTION PLANNING}

In this subsection, aggregate production planning in a production/assembly facility of a consumer electronics supply chain is considered. The production/assembly facility can be modeled as a flexible flowshop that consists of several processing stages in series, with parallel batch machines and input and output buffers of limited capacity, respectively, for holding purchased materials (e.g., product-specific printed wiring boards) waiting for processing and for holding the completed products (e.g., different types of cellular phones) waiting for delivery to the customers (e.g., carriers). Typically, the capacity of input and output buffers is not large enough to limit materials and finished product inventory and to limit the early supplies of purchased materials before their processing dates and early completion of customer orders before the customer-required shipping dates.

The processing stages are as follows: 1) the material preparation stage, where all materials required for the assembly of each product are prepared; 2) postponement stage, where the products for some orders are customized; 3) flashing/flexing stages, where required software is downloaded; and 4) packing stage, where the products and required accessories are packed for shipping. One of the basic goals of aggregate production planning is to maximize the customer service level; that is, to maximize the fraction of customer orders fulfilled on time. A typical customer due-date-related performance measure is the minimization of the number of delayed orders; e.g., Sawik (2007). Simultaneously, increasing competition forces manufacturers to achieve low unit costs by highly and evenly utilizing its renewable production resources (e.g., machines and people) and by minimizing the inventory. Both the input inventory of the purchased materials waiting for processing and the output inventory of the finished products waiting for delivery to the customers should be minimized. These can be achieved by maximizing the number of orders assigned on the due dates and for early or late orders by minimizing their earliness and lateness, respectively, with respect to the due dates. Minimization of the earliness of early orders reduces the output inventory of finished products waiting for delivery to the customers, whereas minimization of the delay of late orders reduces the input inventory of the purchased materials waiting for processing. The aggregate production scheduling problem can be modeled as a dual- or multi-objective time-indexed MIP. The primary scheduling objective is to allocate a set of customer orders with various due dates among planning periods to minimize the number of delayed orders, and the secondary objectives are to level the total input and output inventory, aggregate production, or total capacity utilization over a planning horizon, with limited earliness and lateness for early and late orders, respectively. The following two types of customer orders can be considered:

- small size (single-period) indivisible orders, where each order can be fully processed during a single time period,

- large size (multi-period) divisible orders, where each order cannot be completed in one period and must be split up and processed in more than one consecutive time periods. 
A two-level approach to bi-objective aggregate production planning is considered. The objective of the top-level problem is to determine an assignment of customer orders to planning periods over the horizon to minimize the number of late orders. Given the subsets of delayed orders, the objective of the base-level problem is to allocate customer orders among planning periods to level the aggregate production (or the total capacity utilization) over the horizon. The following four basic decisions are made using the MIP approach:

Order to period assignment, where each customer order is assigned to exactly one planning period.

Order allocation, where, for each multi-period customer order, its allocation among consecutive planning periods is determined.

Order non-delayed/delayed status, where, for each customer order, its completion period is selected with respect to the due date.

Capacity allocation, where the assignment of customer orders to machines is determined.

The top- and base-level MIP models for bi-objective aggregate production scheduling have the following structures, respectively; e.g., Sawik (2006):

Minimize Number of late orders

subject to

1) Order to period assignment constraints, to ensure that each single-period customer order is assigned to exactly one period and each multi-period order is assigned to two or more consecutive planning periods.

2) Order allocation constraints, to ensure that each multi-period customer order is completed.

3) Capacity constraints, to ensure that the demand on capacity at each processing stage is not greater than the available capacity during each period.

4) Input- and output-buffer-capacity constraints, to ensure that, in each period, the total inventory of materials waiting for processing and finished products waiting for shipping to customers does not exceed the input and output buffer capacity, respectively.

Minimize Maximum aggregate production level

or

Maximize Minimum aggregate production level

subject to

1) Order to period assignment constraints, to ensure that the number of late orders is at minimum.

2) Production leveling constraints, to ensure that, during each period, the aggregate production cannot exceed the maximum level to be minimized or cannot be less than the minimum level to be maximized. 
The first objective function of the base-level problem tends to level the aggregate production from above while leaving the lowest production volume close to zero, whereas the second objective function aims at leveling the aggregate production from below while the highest volume is constrained by the maximum-available processing and buffer capacity.

\subsection{COORDINATED PLANNING OF SUPPLIES AND PRODUCTION}

Most work on coordinated supply-chain planning focuses on coordinating the flows of supply and demand over a supply chain to minimize inventory, transportation, and shortage costs. There is a growing body of literature regarding deterministic models on integrated production-distribution planning and scheduling (however, without the supply operations, which are mostly considered separately). A comprehensive review and classification of the existing deterministic models that integrate production and outbound distribution operations at the detailed scheduling level were presented in Chen (2010).

This subsection deals with coordinated medium-term planning of manufacturing and supply of parts and assembly of the finished products in a customer-driven supply chain with a single supplier and single producer. The supply chain consists of the supplier stage (made up of parallel production lines), the producer stage which is a flexible assembly line made up of several assembly stages in series, and a set of customers. The supplier manufactures and delivers product-specific materials (parts) to the producer, where the finished products are assembled according to customer orders and then delivered to the customers to meet their demand. During each period, at most one part type can be manufactured on each production line. When a production line switches from one part type to another, a start-up time should be considered at the beginning of the period. The overall problem is how to coordinate the manufacturing and supply of parts and the assembly of products with respect to the limited capacities and required customer service levels such that the total supply-chain inventory holding cost (or maximum total inventory level) and the production line start-up costs at the supplier stage and part-shipment costs to the producer are minimized. Two decision-making approaches can be applied: an integrated (monolithic) approach, where the coordinated manufacturing, supply, and assembly plans are determined simultaneously; and a hierarchical approach, where first, the assignment of customer orders over the planning horizon and thereby the finished-product-assembly plan is determined, and then the part manufacturing and supply schedules are found. Four basic decisions are made using the MIP approach:

Order to period assignment, where each customer order is assigned to exactly one planning period.

Supplier capacity allocation, where set-ups of production lines for manufacturing different part types are determined over the planning horizon.

Manufacturing lot-size scheduling, where manufacturing lots for each part type are determined at the supplier over the planning horizon.

Supply lot-sizing scheduling, where transportation lots for shipping parts from the supplier to the producer are determined over the planning horizon. 
The multi-objective, time-indexed MIP for coordinated scheduling of manufacturing, supply and assembly has the following structure (Sawik, 2009):

Minimize Total supply chain cost of inventory holding, production line start-ups and material shipment

or

Minimize The weighted sum of the maximum level of total inventory, the number of start-ups and the number of material shipments

\section{subject to}

1) Order to period assignment constraints.

2) Part-type to production line assignment constraints, to ensure that the number of production lines selected for the manufacturing of each part type during each period is not greater than the number of available production lines, given the assignment of the production lines in the previous period.

3) Part manufacturing and shipping constraints, to ensure that the parts are supplied during the periods selected for shipping, and the cumulative supplies by each period are not greater than the initial stocks and cumulative manufacturing of this part type.

4) Producer capacity constraints.

5) Part- and product-inventory-balance constraints.

6) Demand for part-satisfaction constraints, to ensure that the cumulative production of each product type during each period is not greater than the initial stocks and cumulative supplies of the required part types.

7) Manufacturing and production coordinating constraints, to ensure that the cumulative production of each product type by each period is not greater than the initial stocks of the required part types and the cumulative manufacturing of these part types at the supplier.

Of the two proposed criteria, minimization of the maximum inventory level leads to leveling over the horizon of manufacturing of parts and line set-ups and start-ups at the supplier. At the same time the supplier and the producer inventories of parts are higher and the shipping lots are larger. The inventories of parts and the shipping lots are smaller for minimization of the total inventory holding cost. In general, the former objective aims at leveling of production at the supplier at the expense of greater fluctuations and a higher inventory of parts and larger shipping lots (Sawik, 2011).

\section{PLANNING AND SCHEDULING UNDER DISRUPTION RISKS}

In the electronics supply chains, material and information flows are vulnerable to disruption risks due to various natural or man-made disasters such as earthquakes, fires, floods, hurricanes or labor strikes, economic crisis, terrorist attack. Such disaster events are occurring more and more frequently in global and lean supply chains. The disaster probability is very low, however their business impact can be very high. For example, the recent disruptions in the electronics supply chains due to the great East 
Japan earthquake of March 11, 2011 and then the catastrophic Thailand flooding of October 2011, where many component manufacturers were concentrated, resulted in huge losses of many Japanese companies, e.g., Park et al. (2013), Fujimoto and Park (2014).

The recent research focuses on stochastic planning and scheduling in supply chains with disrupted flows. A common tool for planning and scheduling in supply chain under disruption risks is stochastic MIP. Stochastic MIP is an exact mathematical modelling approach that allows for the inclusion of uncertainty by probabilistic scenarios of disruption events and for finding the optimal solutions with respect to multiple objective functions (e.g., Sawik, 2013, 2014a, 2014b). Now, the decision variables used in the deterministic models are additionally defined for each disruption scenario, and for each scenario its occurrence probability is assumed to be known ahead of time. Typical objective functions include expected values for the risk-neutral models and Value-at-Risk or Conditional Value-at-Risk for the risk-averse models. Of particular importance is portfolio approach to supply chain disruption management, e.g., supply portfolio introduced by Sawik (2011). Recent research results on stochastic MIP approaches to scheduling in supply chains under disruption risks are reported by Sawik (2016, 2017), where supplies can be disrupted by multi-level local disaster events or all-or-nothing regional disasters. In Sawik (2016), coordinated scheduling of material supplies and production and distribution of finished products subject to all-or-nothing local and regional disasters are studied. Another important field of study is fair and robust planning and scheduling to reach an equitably efficient expected cost and expected service or equitably efficient average and worst-case performance of a supply chain under disruption risks, e.g., Sawik (2014c, 2015a). Finally, it is worth to mention the Ripple effect in supply chains studied by Ivanov, Sokolov and Dolgui (2014), that can consolidate research in supply chain disruption management, similar to the bullwhip effect regarding demand and lead time fluctuations.

\section{CONCLUSIONS}

This paper has discussed various applications of MIP approaches to planning and scheduling in electronics supply chains. The MIP modeling and decision-making approaches described allow the user to determine optimal or near optimal schedules using advanced algebraic modeling languages such as AMPL and commercially available software for MIP such as CPLEX, XPRESS, Gurobi. This makes the decision-making independent on custom made scheduling software. An important field of further study is application of stochastic MIP for the development of contingency plans to balance competitiveness and robustness and efficient recovery policies for fast rebuilding the electronics supply chains after major disruptions, eg., Sawik (2017).

\section{ACKNOWLEDGEMENTS}

This work has been supported by NCN research grant \#DEC-2013/11/B/ST8/04458, and by $A G H \# 11.11 .200 .324$. 


\section{REFERENCES}

Aguirre, A.M., Méndez, A.C., Gutierrez, G., De Prada, C., 2012. An improvement-based MILP optimization approach to complex AWS scheduling. Computers and Chemical Engineering, 47, pp. 217-226.

Bilyk, A., Mönch, L., Almeder, C., 2014. Scheduling jobs with ready times and precedence constraints on parallel batch machines using metaheuristics. Computers and Industrial Engineering, 78, pp. 175-185.

Chiang, T.C., Fu, L.C., 2012. Rule-based scheduling in wafer fabrication with due date-based objectives. Computers and Operations Research, 39, pp. 2820-2835.

Chen, Z.L., 2010. Integrated production and outbound distribution scheduling: Review and extensions. Operations Research, 58(1), pp. 130-148.

Fujimoto, T., Park, Y.W., 2014. Balancing supply chain competitiveness and robustness through "virtual dual sourcing": lessons from the Great East Japan Earthquake. International Journal of Production Economics, 147, pp. 429-436.

Ivanov, D., Sokolov, B., Dolgui, A., 2014. The Ripple effect in supply chains: trade-off 'efficiency-flexibility-resilience' in disruption management. International Journal of Production Research, 52(7), pp. 2154-2172.

Mönch, L., Fowler, J.W., Mason, S., 2012. Production Planning and Control of Semiconductor Wafer Fabrication Facilities: Modeling, Analysis, and Systems. New York, Springer.

Park, Y., Hong, P., Roh, J.J., 2013. Supply chain lessons from the catastrophic natural disaster in Japan. Business Horizon, 56, pp. 75-85.

Sawik, T., 2001. Mixed integer programming for scheduling surface mount technology lines. International Journal of Production Research, 39, pp. 3219-3235.

Sawik, T., 2002. Balancing and scheduling of surface mount technology lines. International Journal of Production Research, 40, pp. 1973-1991.

Sawik, T., 2006. Hierarchical approach to production scheduling in make-to-order assembly. International Journal of Production Research, 44, pp. 801-830.

Sawik, T., 2007. A lexicographic approach to bi-objective scheduling of single-period orders in make-to-order manufacturing. European Journal of Operational Research, 180, pp. 1060-1075.

Sawik, T., 2009. Coordinated supply chain scheduling. International Journal of Production Economics, 120, pp. 437-451.

Sawik, T., 2011. Scheduling in Supply Chains Using Mixed Integer Programming. Hoboken NJ, Wiley.

Sawik, T., 2013. Integrated selection of suppliers and scheduling of customer orders in the presence of supply chain disruption risks. International Journal of Production Research, 51(23-24), pp. 7006-7022.

Sawik, T., 2014a. Joint supplier selection and scheduling of customer orders under disruption risks: Single vs. dual sourcing. Omega, 43(2), pp. 83-95.

Sawik, T., 2014b. Optimization of cost and service level in the presence of supply chain disruption risks: Single vs. multiple sourcing. Computers \& Operations Research, 51(1), pp. 11-20.

Sawik, T., 2014c. On the robust decision-making in a supply chain under disruption risks. International Journal of Production Research, 52, pp. 6760-6781. 
Sawik, T., 2015a. On the fair optimization of cost and customer service level in a supply chain under disruption risks. Omega, 53, pp. 58-66.

Sawik, T., 2015b. Integrated supply chain scheduling under multi-level disruptions. IFACPapersOnLine, 48(3), pp. 1515-1520.

Sawik, T., 2016. Integrated supply, production and distribution scheduling under disruption risks. Omega, 62, pp. 131-144.

Sawik, T., 2017. A portfolio approach to supply chain disruption management. International Journal of Production Research, 55(7), pp. 1970-1991. 